Conclusions: We observed 25-OHVitamin D levels and bone metabolism biomarkers correction during the first two years after LT. Medical intervention prior to LT as well as antiresorptive treatment seem to play a decisive role in bone mineral density improvement.

Disclosure of Interest: None declared

DOI: 10.1136/annrheumdis-2017-eular.5855

\section{AB0830 BONE MINERAL DENSITY IN MULTIPLE MYELOMA: 39 CASES}

Z. Saoussen, $\underline{H}$. Mouanaa, A. Arfa, M. Jguirim, B. Ismaiel, M. Touzi, N. Bergaoui. Rheumatology departement of Monastir University Hospital, Monastir, Tunisia

Background: In multiple myeloma (MM), osteolysis affects more than $80 \%$ of patients. This leads to bone pain, pathological fractures and hypercalcemia. These lesions result from an alteration of bone remodeling by increased osteoclast activity and decreased osteoblasts one. The real impact of this osteolysis on bone mineral density remains largely understudied. To the best of our knowledge the impact of MM on bone mineralization was studied worldwide only for 6 times where our study is the second biggest one.

Objectives: The aim of the study was to evaluate bone mineralization in the patients with multiple myeloma according to the criteria of diagnostic (IMWG: International Myeloma Working Group 2014, during a period of 5 years (20112016).

Methods: This is a transverse and descriptive study. The bone mineral density was measured by dual-energy X-ray absorptiometry with Lunar Prodigy in spine (L2-L4) and femoral neck.

Results: Thirty-nine patients were collected. The average age was $63 \pm 10$ years [50 years, 86 years] upon them 26 men and 13 women. The sex-ratio is equal to 2 . 11 patients were smoking (35\%), 9 of them had diabetes and only 2 were alcoholic (6\%). The Body Mass Index (BMI) average was $29 \mathrm{~kg} / \mathrm{m}^{2}$. Only one case was underweight $(3 \%)$. The reason of seeking health carewas poor general state in 14 cases $(49 \%)$, bone pain in 22 cases $(78 \%), 5$ cases among them of generalized bone pain $(23 \%)$ and 12 cases of rachialgia $(4.5 \%)$ and only 4 cases of pathological fracture $(15 \%)$. The distribution of patients according to the Durie and Salmon Classification was as follows: 25 cases $(84 \%)$ in stage III, 3 cases $(10 \%)$ in stage II, 2 cases (7\%) in stage I, and 27 cases $(90 \%)$ Type A and 3 cases $(10 \%)$ type B. The average of the monoclonal spike was $34 \mathrm{~g} / \mathrm{L}[2.5 \mathrm{~g} / \mathrm{L}, 88 \mathrm{~g} / \mathrm{L}]$ The heavy chains antibodies were lg $\mathrm{G}$ type in 19 cases $(64 \%)$, IgA type in 7 cases $(24 \%)$, IgM type in only one case (4\%) and IgD type in only one case (4\%). The light chains were Kappa type in 19 cases $(64 \%)$ and Lambda type in 11 cases $(37 \%)$. The ISS score was equal to 1 in 6 cases (23\%), equal to 2 in 13 cases (34\%) and equal to 3 in 8 cases $(30 \%)$. The average bone mass in the spine was $0.998 \pm 0.254 \mathrm{~g} / \mathrm{cm}^{2}$ $\left[0.632 \mathrm{~g} / \mathrm{cm}^{2} ; 1.892 \mathrm{~g} / \mathrm{cm}^{2}\right]$ and in the femoral neck $0.869 \pm 0.254 \mathrm{~g} / \mathrm{cm}^{2}\left[0.632 \mathrm{~g} / \mathrm{cm}^{2}\right.$; $\left.1.892 \mathrm{~g} / \mathrm{cm}^{2}\right]$. The average of the Z-score in the spine was $-0.762 \pm 1.895[-4.4$; $5.7]$ and in the femur $-0.438 \pm 0.962[-2.8 ; 1.4]$. The mean T-score in the spine was $-1.626 \pm 2.025[-4.9 ; 5.6]$ and in the level of the femur $-1.567 \pm 1.178[-3.7 ; 1]$. There was a decrease of bone mineral density noticed in 15 patients (39\%) in at least one place (T-score more than 2.5 SD below normal of young healthy persons. Seventeen patients $(58 \%)$ were candidates for autogenous bone graft. They had induction chemotherapy (Dexamethasone-thalidomide). Others was treated by MPT protocol (Prednisone-Thalidomide-Dexamethasone) in 8 cases (28\%), CDT protocol (Cyclophosphamide-Thalidomide-Dexamethasone) in one case (4\%) and MP protocol (Melphalan -Prednisone) for the remaining (10\%).

Conclusions: $\mathrm{BMD}$ analysis suggests that $\mathrm{MM}$ is associated with systemic bone disease with progressive loss of bone mass at both the spinal and lumbar levels. In order to better study the impact of multiple myeloma and chemotherapy on bone densitometry, a densitometry controlin about 5 years is favorable.

Disclosure of Interest: None declared

DOI: 10.1136/annrheumdis-2017-eular.6754

\section{AB0831 FRAX SCORE: AN INTERESTING WAY FOR GASTROENTEROLOGISTS TO ASSESS FRACTURE RISK IN PATIENTS WITH LONG-TERM PROTON PUMP INHIBITORS}

H. Romdhane ${ }^{1}$, M. Cheikh ${ }^{1}$, K. Abdelghani ${ }^{2}$, R. Ennaifer ${ }^{1}$, N. Bel Hadj ${ }^{1}$, A. Laatar ${ }^{2} .{ }^{1}$ Gastroenterology and hepatology; ${ }^{2}$ Rhumatology, Mongi Slim Hospital, TUNIS, Tunisia

Background: Proton pump inhibitors (PPI) are effective in many indications. Nevertheless, some serious adverse effects associated with prolonged exposure, including an increase in fracture risk, have occurred. This would be explained by two main mechanisms: decreased absorption of calcium secondary to decreased gastric acidity and inhibition of proton pump of osteoclasts reducing bone resorption.

The Frax score assess the 10-year probability of osteoporotic fracture or hip fracture in subjects older than 40 years.

Objectives: The aim of our study was to evaluate the usefulness in our practice of this score in patients under long-term PPI.

Methods: We included patients who had been taking PPI for at least one year. In all patients, we specified the indication and duration of PPI. We then looked for the main personal or family risk factors for osteoporosis. Bone mineral density (BMD) was performed in all patients and frax score was calculated for those older than 40 years.
Results: Fifty-two patients were included in our study. The mean age was $49.5 \pm 14.55$ years [21 - 84 years] with a sex ratio of 0.48 . Long-term PPI were indicated in $75 \%(n=39)$ of patients for gastroesophageal reflux, in $11.5 \%(n=6)$ for chronic gastritis with failure of Helicobacter Pylori eradication, in $3.8 \%(n=2)$ for persistent epigastralgia, in $5.7 \%(n=3)$ for functional dyspepsia and finally in $3.8 \%(n=2)$ of patients in prophylaxis of gastroduodenal lesions in chronic use of NSAIDs. The mean duration of intake was 45.4 months [12-240 months]. The main osteoporotic risk factors were tobacco in $25 \%$, alcohol in $12 \%$, physical inactivity in $42 \%$ and dysthyroidism in $6 \%$ of cases. In our study, 20 women among the 35 included (57\% of cases) were already menopausal. An osteoporotic fracture in a first-degree relative was noted in $23 \%$ of cases, including one parent reporting two fractures. A history of fragility fracture was observed in 11 patients $(21 \%)$ including 3 men and 8 women. The mean daily calcium intake was $567.2 \pm 327.6 \mathrm{mg} / \mathrm{d}$ [230 $-2315 \mathrm{mg} / \mathrm{d}]$. Calcium intake was insufficient in $94 \%$ of patients. BMD was normal in 15 patients $(29 \%$ of cases) while $71 \%(n=37)$ had low BMD. In our population, age $(p=0.02)$, calcium intake $(p=0.029)$ and menopause $(p<0.0001)$ were significantly related to low BMD. The duration of PPI intake was negatively correlated with BMD. Patients taking PPI for at least 30 months were 6.5 times more likely to have low BMD (95\% Cl [1.5-27.4]). The mean FRAX score for major osteoporotic fractures in 37 patients older than 40 years was $1.08 \pm 0.84 \%[0.3-3.3 \%]$. For hip fractures, the mean score was $0.26 \pm 0.29 \%[0-1.4 \%]$. There was a significant correlation between mean Frax score and BMD $(p<0.0001)$. None patient had a patent or subclinical fracture during the follow-up period.

Conclusions: Our study shows an increased risk of fracture in patients under long-term PPI, especially if they have other osteoporotic risk factors. In this context, Frax score is a simple and non-invasive tool for assessing fracture risk in these patients and to adapt screening strategy for sub-clinical fractures. References:

[1] none.

Acknowledgements: none

Disclosure of Interest: None declared

DOI: 10.1136/annrheumdis-2017-eular.5399

\section{AB0832 EFFECT OF LONG-TERM PROTON PUMP INHIBITORS ON BONE MINERAL DENSITY}

H. Romdhane, M. Cheikh, H. Ben Nejma, R. Ennaifer, N. Bel Hadj. Gastroenterology and hepatology, Mongi Slim Hospital, TUNIS, Tunisia

Background: Proton pump inhibitors (PPI) are widespread nowadays. Recent concerns have emerged about possible bone complications of long-term use of PPIs, such as low bone mineral density (BMD) and an increased risk of fractures. Objectives: The aim of our study was to evaluate the effect of long-term use of PPIs on bone by measuring the BMD in order to estimate the frequency of osteopenia and osteoporosis, and to determine the risk factors associated to this complication.

Methods: It was a prospective study including consecutive patients who where taking proton pump inhibitors for at least one year. In all patients we realized a bone densitometry in order to evaluate the bone strength and we calculated the FRAX score to estimate the risk of osteoporotic fracture at ten years.

Results: We included 52 patients. The mean age was 49,5 years old. The malefemale ratio $\mathrm{M} / \mathrm{F}$ was 0,48 . At least three risk factors were found in more than $50 \%$ of the population. The calculated daily calcium intake was insufficient in $94 \%$ of the patients. The mean duration of PPIs intake was 45 months. The most frequent indication was gastro esophageal reflux disease $(75 \%)$. The PPI prescription was appropriate in $94 \%$ of the cases. The prevalence of osteopenia and osteoporosis was respectively $52 \%$ and $19 \%$. The predictive factors of low BMD were an age $\geq 50$ years old $(p=0,03)$, the menopause $(p<0,0001)$, a calcium intake $\leq 550 \mathrm{mg} /$ day $(p<0,038)$, and a PPI use duration $\geq 30$ months $(p<0,006)$. The multivariate study could not be undertaken because of co linearity of the factors. Conclusions: The long term PPI use is associated to the risk of bone complications, especially among patients at risk for osteoporosis. It seems reasonable to be more vigilant in prescribing PPIs and use lowest effective dose for patients with appropriate indications, and to screen these complications if necessary.

Disclosure of Interest: None declared

DOI: 10.1136/annrheumdis-2017-eular.5281

\section{AB0833 BONE MINERAL DENSITY IN TUNISIAN PATIENTS WITH AUTOIMMUNE HEPATITIS}

M. Cheikh, H. Romdhane, H. Bennejma, R. Ennaifer, N. Bel Hadj. Gastroenterology and hepatology, Mongi Slim Hospital, TUNIS, Tunisia

Background: Bone loss in autoimmune hepatitis (AlH) is scanty and conflicting. The pathogenic mechanisms are not completely elucidated.

Objectives: This study aimed to assess the prevalence and risk factors for bone loss in patients with AlH.

Methods: Bone mineral density (BMD) using X-ray absorptiometry at both lumbar spine and femoral neck sites was measured in patients with AlH. Were excluded patients with diseases disturbing the bone density. Osteopenia was considered if T-score $<-1.5 \mathrm{DS}$ and osteoporosis if T-score $<-2.5 \mathrm{DS}$. 
Results: Twenty eight patients were enrolled in the study. They were 19 women (sex-ration $\mathrm{M} / \mathrm{F}=0.6$ ), with a mean age of 54 years [extremes: 13 - 73 years]. Most patients had type $1 \mathrm{AlH}(89.2 \%)$. Seventeen patients were diagnosed at stage of cirrhosis $(60.7 \%)$. Associated auto-immune manifestations were observed in $42.8 \%$ of cases. Overlap syndrome with primary biliary cirrhosis was noted in $21.4 \%$ of cases. Fifty five percent of patients were on steroid treatment with or without azathioprine. BMD was low in 9 patients $(32 \%)$ as fellow: osteopenia in 6 cases and osteoporosis in 3 cases. There was a correlation between bone loss and use of steroid treatment but it wasn't statistically significant $(p=0.07)$.

Conclusions: In our series, the prevalence of bone loss in AlH is high (45\%). This data suggests that bone status should be assessed routinely in patients with $\mathrm{AlH}$, especially in those on steroid treatment.

Disclosure of Interest: None declared

DOI: 10.1136/annrheumdis-2017-eular.6442

\section{AB0834 RISK FACTORS FOR DECREASED BONE MINERAL DENSITY IN INFLAMMATORY BOWEL DISEASE IN A TUNISIAN COHORTE}

H. Romdhane ${ }^{1}$, M. Cheikh ${ }^{1}$, K. Abdelghani ${ }^{2}$, R. Ennaifer ${ }^{1}$, N. Bel Hadj ${ }^{1}$, A. Laatar ${ }^{2} .{ }^{1}$ Gastroenterology and hepatology; ${ }^{2}$ Rhumatology, Mongi Slim Hospital, TUNIS, Tunisia

Background: Patients with inflammatory bowel disease [IBD] are at risk for metabolic bone disease. Many studies have identified various risk factors but most of them have involved western patients.

Objectives: The aim of this study was to investigate the prevalence and the risk factors for metabolic bone disease in Tunisian IBD patients.

Methods: Retrospective study including patients with IBD admitted in our department between January 2011 and December 2015. Demographic and clinical characteristics of patients were analysed. Bone mineral density of the femoral neck, total femur and lumbar spine was quantified by dual-energy X-ray absorptiometry.

Results: Among 82 patients followed for IBD $(70.7 \%$ with Crohn's disease; $29.3 \%$ with Ulcerative colitis), a bone densitometry was performed in $56 \%$ of cases $(n=46) .16$ patients have osteopenia and 7 had osteoporosis, as assessed by T-score. Univariate analysis showed that Crohn'n disease in particular ileal disease, high steroid dose and the presence of extra-intestinal manifestations were significantly associated with a low bone mineral density (for all $p<0.05$ ). In the other hand, IBD duration since diagnosis, sexe, tabagism were not associated with bone loss.

In multivariate regression analysis, risk factors for decreased bone mineral density were IBD duration since diagnosis, high steroid dose, ileal crohn's disease and extra-intestinal manifestations.

Conclusions: In our Tunisian cohort of IBD patients, Crohn's disease, high steroid dose and extra-intestinal manifestations were associated with increased risk for metabolic bone disease. High risk patients should be identified and appropriate therapies should be started early to improve long term quality of life.

Disclosure of Interest: None declared

DOI: 10.1136/annrheumdis-2017-eular.5301

\section{AB0835 DENOSUMAB AS A FIRST CHOICE DRUG FOR GLUCOCORTICOID INDUCED OSTEOPOROSIS TREATMENT INSTEAD OF BISPHOSPHONATE}

I. Yoshii ${ }^{1}$, T. Chijiwa ${ }^{2} .{ }^{1}$ Orthopaedic Surgery, Yoshii Hospital, Shimanto City; ${ }^{2}$ Rheumatology, Kochi Memorial Hopsital, Kochi, Japan

Background: Glucocorticoid induced osteoporosis (GIO) is serious problem for raising risk of bone fragile fracture. In general, first choice drug for $\mathrm{GIO}$ is bisphosphonate (BPH), however, denosumab (dMAB), a monoclonal antibody of receptor activator of nuclear factor kappa-B ligand, is closed up as a alternative selection for $\mathrm{GIO}$ recently.

Objectives: The aim of this study is to evaluate effectiveness of dMAB in bone mineral density (BMD) for GIO treatment compared to $\mathrm{BPH}$.

Methods: In the patients in whom glucocorticoid steroid (GCs) have been administered for more than three months, who met indication criteria for GIO what was determined by the Japanese Society for Bone and Mineral Research in 2014, that is matrix calculated in adding points of past fracture history, age, dosage of GCs, and BMD value (1), were enrolled. Before March 2013, data was lacking, so patients who have been administrated GCs after April 2013 were picked up. Patients BMD at GCs administration, at 6 months after initial treatment, if drug was changed, also at 6 months after second treatment, for minimum lumbar spine (LS), femoral neck (FN), and greater trochanter (GT) were measured with dual-energy X-ray absorptiometry (DEXA). Patients were classified by drug for initial treatment and second drug if administered. Patients age, initial, average, and total dose of GCs, term length of administration, and BMD and its gain for each chance were compared with Mann-Whitney U-test and Student's paired T-test.

Results: 149 patients in whom 48 with no drug administrated (N), 24 for BPH naïve and continued (BB), 22 for $B P H$ naïve and changed to $\mathrm{dMAB}(\mathrm{BD}), 21$ for $\mathrm{DMAB}$ naïve and continued (DD), 34 for dMAB naïve and changed to $\mathrm{BPH}$ (DB) were counted. In these, sex distribution was 26 for men and 123 for women. Underlying disease for administration of GCs were rheumatoid arthritis for 114 , polymyalgia rheumatica for 12 , idiopathic thrombocytopenic purpura for
9, systemic lupus erythematosus for 6 , and others for 8 . For groups, age at baseline, initial, average, and total dose, and term length of administration of GCs demonstrated no significant difference between any pairs of the groups. BMD at baseline for Group N demonstrated significant greater per-cent of young adult mean (\%YAM) than Group DD $(p<0.01)$ in all parts, yet greater than the other groups but not statistically significant. In Group N, BMD had significantly decreased from the baseline to 6 months later in all parts $(p<0.01)$. In the other groups, BMD had shown gain at 6 months after drug administration in all part, however, in Group DB showed mean \%YAM loss for GT after first and second drug administration compared to Group BD had shown \%YAM loss after first but gain after second drug for FN (Table 1).

\begin{tabular}{|c|c|c|c|c|c|}
\hline First Drug (FD) & none & \multicolumn{2}{|c|}{ bisphosphonate } & \multicolumn{2}{|c|}{ denosumab } \\
\hline Second Drus (SD) & none & none & denosumab & none & bisphosphonate \\
\hline Cases & 48 & 24 & 22 & 21 & 34 \\
\hline age at baseline & $69.7 \pm 10.8$ & $73.7 \pm 16.5$ & $81.8 \pm 122$ & $72.5 \pm 11.5$ & $71.5 \pm 10.8$ \\
\hline initial dose of GCs & $6.78 \pm 8.98$ & $6.88 \pm 8.25$ & $5.58 \pm 6.95$ & $5.21 \pm 3.47$ & $6.95 \pm 2.25$ \\
\hline average dose of GCs & $3.77 \pm 2.13$ & $5.49 \pm 3.76$ & $5.06 \pm 1.61$ & $3.49 \pm 1.49$ & $5.12 \pm 2.06$ \\
\hline term length of administration & $53.6 \pm 45.3$ & $84.7 \pm 44.9$ & $38.2 \pm 46.4$ & $51.9 \pm 58.7$ & $65.2 \pm 46.0$ \\
\hline total dose of $\mathrm{GCs}_{\mathrm{s}}$ & $3836.6 \pm 4444.3$ & $7170.2 \pm 10923.8$ & $2447.1 \pm 6365.9$ & $5017.1 \pm 9057.3$ & $7898.2 \pm 772.9$ \\
\hline BMD of lumbar spine at baseline & $89.1 \pm 16.9$ & $78.1 \pm 17.7$ & $80.2 \pm 124$ & $70.9 \pm 9.3$ & $75.9 \pm 12.4$ \\
\hline BMD of LS at six months after administration of FD & $86.5 \pm 19.1$ & $79.3 \pm 10.5$ & $81.8 \pm 15.2$ & $72.9 \pm 13.5$ & $75.9 \pm 15.5$ \\
\hline BMD of LS at six months after administration of SD & $2<$ & $2<$ & $81.2 \pm 12.8$ & $2<$ & $77.3 \pm 9.2$ \\
\hline BMD of FN at baseline & $90.8 \pm 12.8$ & $77.3 \pm 9.3$ & $70.6 \pm 12.9$ & $66.8 \pm 7.6$ & $72.1 \pm 8.8$ \\
\hline $\mathrm{BMD}$ of $\mathrm{FN}$ at six months after administration of $\mathrm{FD}$ & $87.8 \pm 15.4$ & $80.0 \pm 14.6$ & $69.1 \pm 122$ & $65.9 \pm 12.4$ & $74.3 \pm 11.4$ \\
\hline BMD of $F N$ at six months after administration of SD & $2<$ & 2 & $71.2 \pm 11.3$ & $>$ & $74.4 \pm 15.3$ \\
\hline BMD of GT at baseline & $85.8 \pm 9.8$ & $80.8 \pm 10.1$ & $71.2 \pm 12.7$ & $67.1 \pm 12.6$ & $75.2 \pm 9.4$ \\
\hline BMD of GT at six months after administration of FD & $83.5 \pm 14.5$ & $80.1 \pm 12.9$ & $71.2 \pm 12.7$ & $68.9 \pm 12.6$ & $75 \pm 9.3$ \\
\hline$B M D$ of $G T$ at six months after administration of SD & $\sum<$ & $\sum<$ & $74.2 \pm 13.3$ & $\sum<$ & $74.2 \pm 10.3$ \\
\hline
\end{tabular}

Conclusions: From these results, dMAB is effective role in raising BMD for GIO as a initial drug, and a second drug even after inadequate response to $\mathrm{BPH}$. dMAB could be possible to be chosen as a first choice drug for GIO treatment. References:

[1] Suzuki, Y., Nawata, H., Soen, S. et al. J Bone Miner Metab (2014) 32: 337. doi:10.1007/s00774-014-0586-6.

Disclosure of Interest: None declared

DOI: 10.1136/annrheumdis-2017-eular.3106

\section{AB0836 PERFORMANCE OF QUANTITATIVE ULTRASOUND AND SIX OSTEOPOROSIS RISK INDEXES IN MENOPAUSAL WOMEN: VALIDATION AND COMPARATIVE EVALUATION STUDY}

I. Ghozlani ${ }^{1}$, A. El Maataoui ${ }^{2}$, M. Ghazi ${ }^{3}$, A. Kherrab ${ }^{3}$, R. Niamane ${ }^{3}$. ${ }^{1}$ Rheumatology Department, Military Hospital; ${ }^{2}$ Chemistry-Biochemistry Department, Faculty of Medicine and Pharmacy, Ibn Zohr University, Agadir; ${ }^{3}$ Rheumatology Department, Military Hospital Avicenne, Marrakech, Morocco

Background: A number of questionnaire-based systems and the use of portable quantitative ultrasound scanners (QUS) have been devised in an attempt to produce a cost-effective method of screening for osteoporosis.

Objectives: to assess the sensitivity and specificity of different techniques and their ability to act as screening tools in relation to dual energy $\mathrm{X}$-ray absorptiometry (DXA).

Methods: 295 white postmenopausal women aged over 60 were enrolled. Each subject completed a standardized questionnaire which permits the measure of six osteoporosis indexes and had bone mineral density (BMD) measured using QUS and DXA. Sensitivity and specificity of the different techniques in relation to DXA were plotted as receiver-operating characteristic (ROC) curves at DXA T-score total hip $\leq-2.5$ (osteoporosis).

Results: BUA sensitivity and specificity values were respectively $76.8 \%$ and $51.2 \%$ at the total hip. The optimal cut-off T-score for QUS was -2 at the total hip. The osteoporosis self-assessment tool (OST) provided consistently the highest AUC $(0.80)$ among the clinical tools and had the best sensitivity and specificity balance $(90.2 \%>44.5 \%)$. OST negative likelihood ratio was 0.22 .

Conclusions: OST (based only on the weight and the age) performed slightly better than QUS and other risk questionnaires in predicting low BMD at the total hip.

Disclosure of Interest: None declared

DOI: 10.1136/annrheumdis-2017-eular.1449

\section{AB0837 BONE METABOLISM AND OSTEOPOROSIS RISK FACTORS ANALYSIS IN SPINAL CORD INJURY PATIENTS AT TWELVE MONTHS FOLLOW UP}

I. Martínez Cordellat ${ }^{1}$, F. Torralba Collados ${ }^{2}$, C. Grao Castellote ${ }^{2}$, E. Grau García $^{1}$, V. Fornes Ferrer ${ }^{3}$, E. Labrador Sánchez ${ }^{1}$, K. Arévalo Ruales ${ }^{1}$, J. Fragio Gil ${ }^{1}$, R. González Mazarío ${ }^{1}$, C. Alcañiz Escandell ${ }^{1}$, I. Chalmeta Verdejo ${ }^{1}$, C. Feced Olmos ${ }^{1}$, L. González Puig ${ }^{1}$, J. Ivorra Cortés ${ }^{1}$

R. Negueroles Albuixec ${ }^{1}$, J.E. Oller Rodríguez ${ }^{1}$, F. Ortiz Sanjuán ${ }^{1}$, E. Bernabeu $V_{\text {Vicens }}{ }^{1}$, C. Nájera Herranz ${ }^{1}$, I. Cánovas Olmos ${ }^{1}$, J.A. Román Ivorra ${ }^{1}$

${ }^{1}$ Rheumatology Department; ${ }^{2}$ Spinal Cord Injury Unit; ${ }^{3}$ Biostatistics Unit IIS Ia $\mathrm{Fe}$, Hup la Fe, Valencia, Spain

Background: The spinal cord injury associated with the immobilization of the 